\title{
Node Localization of Wireless Sensor Networks Based on DV-hop and Steffensen Iterative Method
}

\author{
Wang Zhi Ping and Chen Xuan \\ (Zhejiang Industry Polytechnic College, Shaoxing, 312000, China) \\ zhejiangwzp@126.com,734140999@qq.com
}

\begin{abstract}
In order to reduce localization error of the sensor node, a novel node localization algorithm (DVHop-Steff) is proposed in this paper based on DV-Hop algorithm and Steffensen iterative method. Firstly, DV-Hop algorithm is used to obtain location position of wireless sensor nodes; and then Steffensen iterative method is used to correct the locate results of DV-Hop algorithm and achieve higher localization precision, finally, the performance of the proposed algorithm is tested by simulation analysis. The results show that the localization precision of the proposed algorithm has been improved significantly and has more wide application scope.
\end{abstract}

Keywords: wireless sensor network, node location, DV-Hop algorithm, Steffensen iterative method

\section{Introduction}

Wireless Sensor Network (WSN) is a self-organizing network system composed of a large number of micro-sensor nodes with sensing and communication capabilities, aiming at sensing and collecting the information of object in the coverage area. In practical applications, the network monitoring without position information is meaningless, so accurate localization of the sensor nodes is the foundation of wireless sensor network applications [1].

Since WSN has great application values, domestic and foreign experts and scholars have carried out a series of studies. The current sensor node localization algorithm consists of distance-dependent algorithm and distance-independent algorithm [2]. Distance-dependent localization algorithm is mainly composed of Received Signal Strength Indicator (RSSI), Time of Arrival (TOA), Time Difference of Arrival (TDOA) and Angle of Arrival (AOA) [3, 4]. Due to great interference caused by environmental factors, the obtained error is relatively large with and high costs. Distance-independent localization algorithm is mainly composed of centroid algorithm and DV-Hop algorithm $[5,6]$. Distance-independent localization algorithm reduces its requirements over node hardware, has strong advantages in costs and power consumption, and the localization accuracy is less affected by environmental factors, so it has become a main research direction. It is relatively simple to implement DV-Hop algorithm. Unknown node localization can be achieved by only requiring a small number of anchor nodes, which has won wide concern but the accuracy is to be improved. Therefore, scholars have proposed some improved DV-Hop algorithm. For example, literature [7] introduced the RSSI strategy to the calculation of DV-Hop algorithm node distance, in order to reduce the inter-node errors and improve the localization accuracy; literature [8] introduced the medium access mechanism to DV-Hop algorithm, in order to adjust the distance error; literature [9] introduced the optimal adjustment factor to correct the distance of each anchor node; in order to reduce the calculation error of the average hop distance.

To reduce the sensor node localization errors, this paper proposed a sensor node localization method (DVHop-Steff) based on DV-Hop Steffensen error correction. This 
paper first adopts the DV-Hop algorithm to roughly localize the wireless sensor nodes; then corrects the DV-Hop algorithm's localization results by adopting the Steffensen iteration method to achieve precise localization, and finally makes simulation test towards the localization performance of DVHop-Steff algorithm.

\section{DV-Hop Algorithm and Error Analysis}

\subsection{DV-Hop Localization Algorithm}

DV-Hop algorithm's specific steps are as follows:

(1) Anchor nodes broadcast packets to neighboring nodes, unknown nodes obtain the minimum hops of each anchor node, and then forward to the neighboring nodes of hops value plus one.

(2) After each anchor node obtaining other nodes' position information and number of hops, the distance of average per-hop can be estimated by using Equation (1).

$$
\text { Hop Size }_{i}=\sum_{j \neq i} \sqrt{\left(x_{i}-x_{j}\right)^{2}+\left(y_{i}-y_{j}\right)^{2}} / \sum_{j \neq i} \text { hop } S_{i j}
$$

Where, (xi,yi) and (xj,yj) are the coordinates of anchor nodes $\mathrm{i}$ and $\mathrm{j}$, and hopsij is the minimum number of hops between $i$ and $j$.

(3) The estimated distance between the unknown node and anchor node is:

$$
L_{i}=S_{i} \times \text { HopSize }
$$

(3) According to the distance between the unknown nodes and each anchor node, use the multilateral method to calculate the coordinates of unknown nodes, as shown in below equation:

$$
\left\{\begin{array}{c}
\left(x_{i}-x_{1}\right)^{2}+\left(y_{i}-y_{1}\right)^{2}=L_{1}^{2} \\
\left(x_{i}-x_{2}\right)^{2}+\left(y_{i}-y_{2}\right)^{2}=L_{2}^{2} \\
\vdots \\
\left(x_{i}-x_{j}\right)^{2}+\left(y_{i}-y_{j}\right)^{2}=L_{j}^{2}
\end{array}\right.
$$

Where, $(x i, y i)$ is the coordinate of unknown node; $(x 1, y 2), \ldots,(x j, y j)$ are the coordinates of anchor nodes recorded by the unknown node.

Given the (j-1)-th equation in Equation (3) subtracts the last equation in order, a linear expression $\mathrm{AX}=\mathrm{b}$ is obtained.

\subsection{DV-Hop Localization Error Analysis}

Affected by many factors, there are errors for DV-Hop algorithm to measure the distance L, so the solution of unknown node coordinate's linear equation group is expressed as follows:

$$
A X+\varepsilon=b
$$

Where, $\varepsilon$ is the random error vector at the $\mathrm{n}-1$-th dimension.

Use the least square method to get the solution of equations. However, each element of b contains Lj with errors, which seriously affect the accuracy of the least squares method [11].

Assumed fn is the distance error between the unknown node and the anchor nodes, then

$$
f_{j}=\sqrt{\left(x_{i}-x_{j}\right)^{2}+\left(y_{i}-y_{n}\right)^{2}}-L_{j}
$$


According to Equation (3), the unknown nodal coordinate satisfy the following equation:

$$
\left\{\begin{array}{c}
f_{1}=\sqrt{\left(x_{i}-x_{1}\right)^{2}+\left(y_{i}-y_{1}\right)^{2}}-L_{1} \\
f_{2}=\sqrt{\left(x_{i}-x_{2}\right)^{2}+\left(y_{i}-y_{2}\right)^{2}}-L_{2} \\
\vdots \\
f_{j}=\sqrt{\left(x_{i}-x_{n}\right)^{2}+\left(y_{i}-y_{j}\right)^{2}}-L_{j}
\end{array}\right.
$$

After solving the unknown nodal coordinate, the following equation is obtained

$$
f\left(x_{i}, y_{i}\right)=\sum_{j=1}^{n}\left(\sqrt{\left(x_{i}-x_{j}\right)^{2}+\left(y_{i}-y_{j}\right)^{2}}-L_{j}\right)
$$

When Equation (11) obtains the minimum value, the total error is minimized, and the solution of unknown node gets closest to the true value. At this time, the coordinate $(x, y)$ is the optimal solution, satisfying the below equation's unknown nodal coordinate:

$$
\text { fitness }\left(x_{i}, y_{i}\right)=\min \left(\sqrt{\left(x_{i}-x_{j}\right)^{2}+\left(y_{i}-y_{j}\right)^{2}}-L_{j}\right)
$$

Through the above method, this paper successfully transforms 1.2 DV-Hop localization error problems into non-linear optimization problems, solves Equation (8) by adopting the Steffensen iteration, and improves the localization accuracy of the sensor.

\section{Steffensen DV-Hop Localization Error Correction}

\subsection{Steffensen Algorithm}

The nonlinear equation $f(x)=0$ can be transformed into equivalent equation:

$$
x=\varphi(x)
$$

Select an initial approximation $x 0$ to build the iteration sequence:

$$
x_{k+1}=\varphi\left(x_{k}\right), k=0,1,2, \ldots
$$

In the equation, $\varphi(x)$ is called iterated function.

Steffensen iteration method is an improved fixed point iteration method with advantages of quadratic convergence speed and calculation accuracy, and the iterated function is:

$$
\varphi(x)=x-\frac{(\varphi(x)-x)^{2}}{\varphi(\varphi(x))-2 \varphi(x)+x}
$$

Steffensen iteration equation is:

$$
\left\{\begin{array}{l}
x_{k}=\varphi\left(x_{k}\right), z_{k}=\varphi\left(y_{k}\right) \\
y_{k+1}=x_{k}-\frac{\left(y_{k}-x_{k}\right)^{2}}{z_{k}-2 y_{k}+x_{k}}, k=0,1,2, \ldots
\end{array}\right.
$$

According to the above equation, Steffensen iteration steps are:

(1) Take the initial point $x 0$, the maximum number of iteration $N$ and precision requirement $\varepsilon$, set $k=0$.

(2) Calculate $y_{k}=\varphi\left(x_{k}\right), \quad z_{k}=\varphi\left(y_{k}\right), \quad y_{k+1}=x_{k}-\frac{\left(y_{k}-x_{k}\right)^{2}}{z_{k}-2 y_{k}+x_{k}}$.

(3) If $\left|x_{k+1}-x_{k}\right|<\varepsilon$, stop the calculation. 
(4) If $k=N$, stop the calculation; otherwise, set $k=k+1$, go to step (2)

\subsection{Steffensen DV-Hop Localization Error Correction}

To obtain the minimum value from Equation (8), the necessary condition is:

$$
\frac{\partial f}{\partial x_{i}}=\frac{\partial f}{\partial y_{i}}=0,(m<i \leq n)
$$

Where:

$$
\left\{\begin{array}{l}
\frac{\partial f}{\partial x_{i}}=\sum_{\substack{m i<\leq \leq n \\
1 \leq j \leq m}}\left(2\left(x_{i}-x_{j}\right)-\frac{2 l_{i j}\left(x_{i}-x_{j}\right)}{\sqrt{\left(x_{i}-x_{j}\right)^{2}+\left(y_{i}-y_{j}\right)^{2}}}\right) \\
\frac{\partial f}{\partial y_{i}}=\sum_{\substack{m<i \leq n \\
1 \leq j \leq m}}\left(2\left(y_{i}-y_{j}\right)-\frac{2 l_{i j}\left(y_{i}-y_{j}\right)}{\sqrt{\left(x_{i}-x_{j}\right)^{2}+\left(y_{i}-y_{j}\right)^{2}}}\right)
\end{array}\right.
$$

Assumed:

$$
\Delta_{i}=\sqrt{\left(\frac{\partial f}{\partial x_{i}}\right)^{2}+\left(\frac{\partial f}{\partial y_{i}}\right)^{2}}
$$

The issue of min(fitness())'s optimal solution is transformed into the issue of unknown nodal coordinate $(x i, y i)$ when solving the minimum value of $\Delta_{i}$. Specific steps are:

(1) According to the first step of Steffensen iteration Equation (12), iterate the variables $x i$ and $y i$, the below equation is obtained:

$$
\begin{aligned}
& \left\{\begin{array}{l}
\bar{x}_{i}=\varphi\left(x_{i}\right)=\frac{\sum_{1 \leq j \leq m}\left(x_{j}+\frac{l_{i j}\left(x_{i}-x_{j}\right)}{\sqrt{\left(x_{i}-x_{j}\right)^{2}+\left(y_{i}-y_{j}\right)^{2}}}\right)}{m} \\
y_{i}=\varphi\left(y_{i}\right)=\frac{\sum_{\mid \leq j \leq m}\left(y_{j}+\frac{l_{i j}\left(y_{i}-y_{j}\right)}{\sqrt{\left(x_{i}-x_{j}\right)^{2}+\left(y_{i}-y_{j}\right)^{2}}}\right.}{m}
\end{array}\right. \\
& \left\{\begin{array}{c}
\sum_{i j}\left(\sum _ { i j } \left(x_{j}+\frac{l_{i j}\left(x_{i}-x_{j}\right)}{\sqrt{\left(x_{i}-x_{j}\right)^{2}+\left(y_{i}-y_{j}\right)^{2}}}\right.\right. \\
x_{i}=\varphi\left(x_{i}\right)=\frac{\sum_{1 s j \leq m}\left(y_{j}+\frac{l_{i j}\left(y_{i}-y_{j}\right)}{\sqrt{\left(x_{i}-x_{j}\right)^{2}+\left(y_{i}-y_{j}\right)^{2}}}\right.}{m}
\end{array}\right.
\end{aligned}
$$

Where, $x i$ and $y i$ adopt the estimated location of the sensor nodes by using DV-Hop algorithm, which is used as the initial iteration.

(2) According to the second step of Equation (12), the update iteration operation is made for the unknown nodal coordinate:

$$
\left\{\begin{array}{l}
x_{i}^{\prime}=\tilde{x}_{i}-\frac{\left(x_{i}-x_{i}\right)^{2}}{\tilde{x}_{i}-2 \bar{x}_{i}+x_{i}} \\
y_{i}^{\prime}=\tilde{y}_{i}-\frac{\left(\bar{y}_{i}-y_{i}\right)^{2}}{\bar{y}_{i}-2 y_{i}+y_{i}}
\end{array}\right.
$$

(3) Substitute new unknown nodal coordinate (xi,yi) into Equation (15) to calculate the 
value of $\Delta_{i}$.

(4) When the number of iteration reaches the set maximum or the iteration precision reaches the set value, stop the iteration, solve the unknown nodal coordinate getting closest to the true value, so that the localization error is minimized.

To sum up, DVHop-Steff localization process is shown in Figure 1.

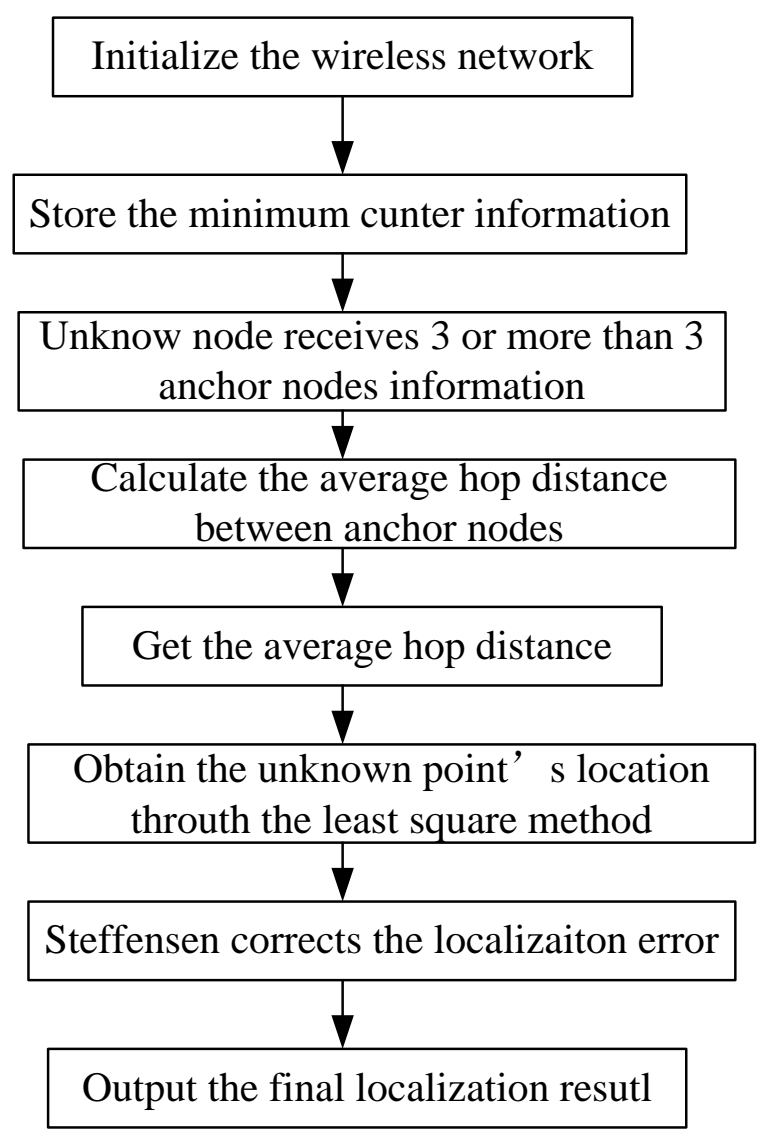

Figure 1. Nodal Localization Process

\section{Simulation Test}

\subsection{Simulation Environment}

Assumed 100 sensor nodes are randomly distributed in a $100 \mathrm{~m} \times 100 \mathrm{~m}$ square area, the nodal communication radius is the same, as shown in Figure 2 . To verify the validity of DVHop-Steff algorithm, this paper implements the simulation test on the Matlab 2012 platform, compares with the traditional DV-Hop algorithm and ADV-Hop algorithm proposed in literature [12], and uses the localization performance of localization error measuring algorithm. Specific definition is as follows:

$$
\text { error }=\frac{\sum_{i=1}^{n-m}\left\|N_{\text {Estimation }}^{i}-N_{\text {Actual }}^{i}\right\|}{n-m}
$$

Where, $n$ and $m$ respectively refer to total number of nodes and number of anchor nodes. 


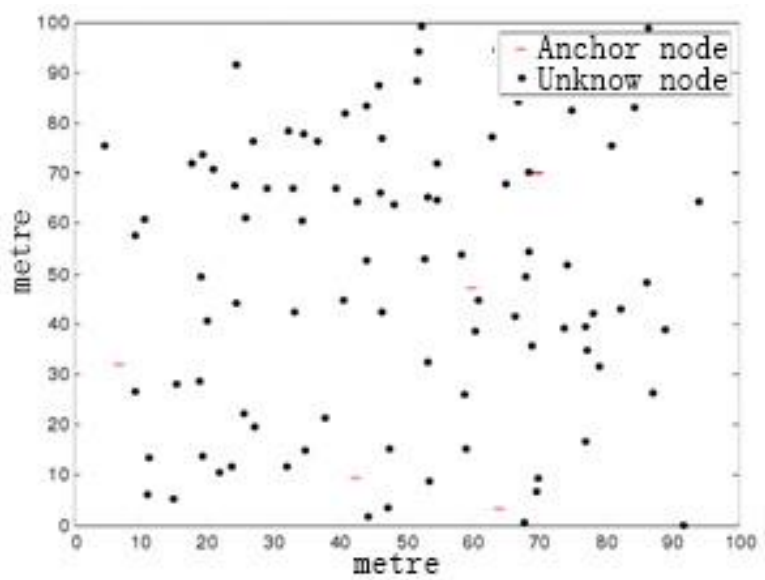

Figure 2. Node Distribution

\subsection{Results and Analysis}

(1)Effects of Ranging Error on Localization Accuracy

Under different ranging errors, the average localization error changing curve of ADV-Hop, DV-Hop and DVHop-Steff algorithms is shown in Figure 3. From Figure 3, compared with DV-Hop algorithm, ADV-Hop and DVHop-Steff algorithms are featured by smaller localization error and higher localization accuracy. Since DVHop-Steff uses Steffensen iteration to further solve DV-Hop algorithm localization results, the more accurate localization results than ADV-Hop algorithm are obtained.

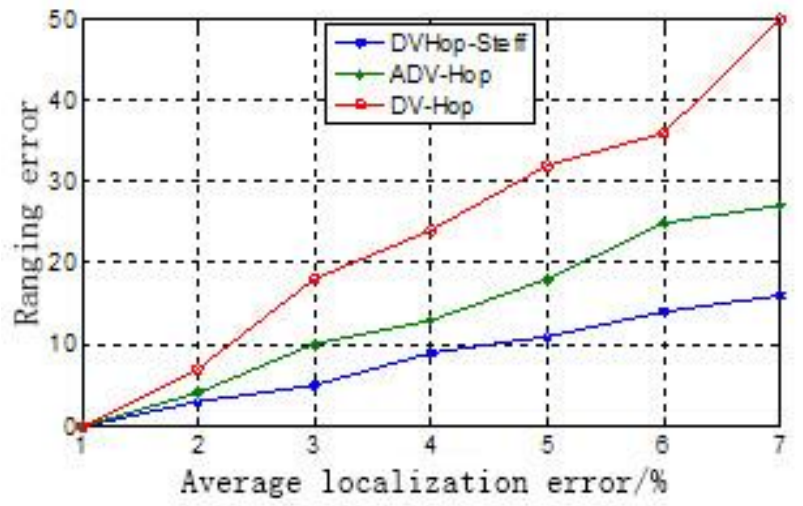

Figure 3. Changes of Ranging Error

(2)Effects of Anchor Nodes on Localization Accuracy

In the different anchor nodes, the average localization error changing curve of ADV-Hop, DV-Hop, DVHop-Steff algorithms is shown in Figure 4. According to Figure 4 , as anchor nodes increase, more accurate localization is expected to obtain when anchor nodes are less. Therefore, compared with the comparison algorithm, DVHop-Steff algorithm has lower costs. In addition, Figure 4 shows that DVHop-Steff localization algorithm may realize more accurate localization in the smaller density of anchor nodes. 


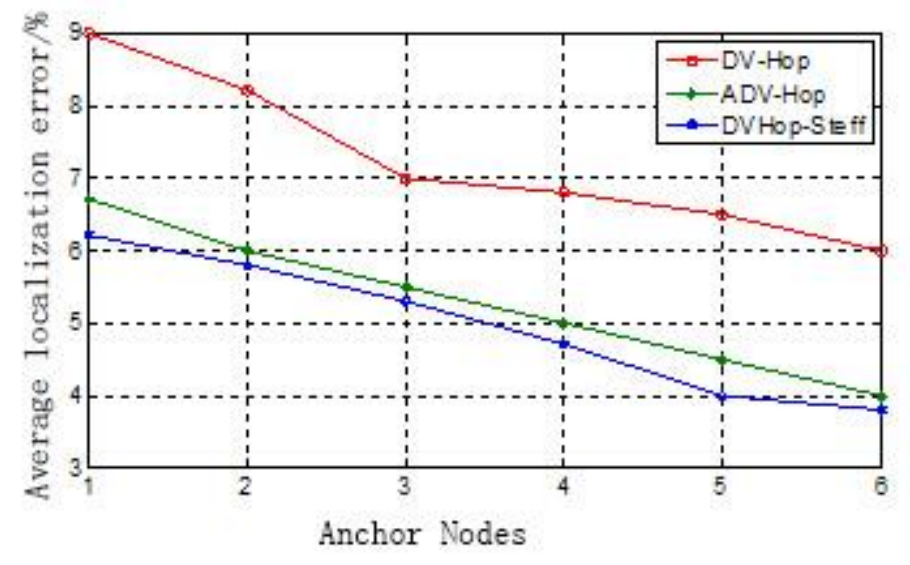

Figure 4. Changes of Anchor Nodes

(3)Effects of Network Connectivity on the Localization Accuracy

In different network connectivity conditions, the average localization error changing curve of ADV-Hop, DV-Hop and DVHop-Steff algorithms is shown in Figure 5. According to Figure 5, compared with DV-Hop localization algorithm, ADV-Hop and DVHop-Steff algorithm can realize more accurate localization. When the measuring radius $R$ is smaller, DVHop-Steff algorithm has more obvious localization advantages over the comparison algorithm.

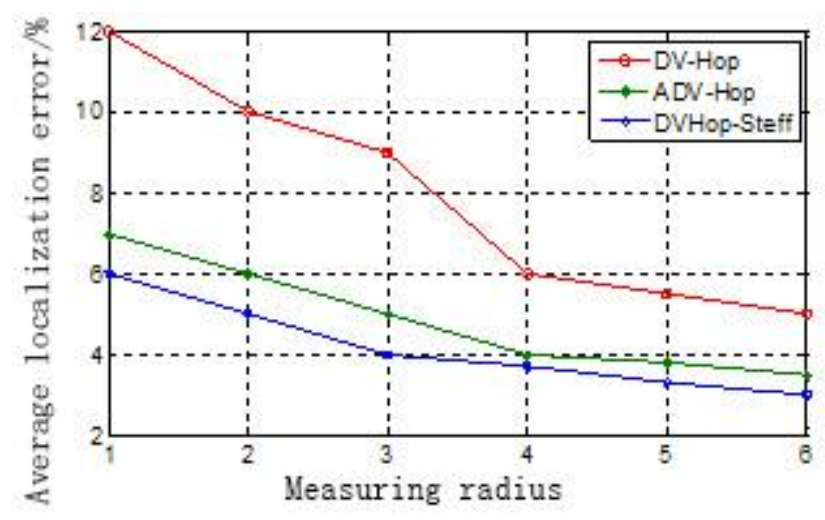

Figure 5. Changes of Network Connectivity

\section{Conclusion}

To solve the existing issues of DV-Hop algorithm, this paper puts forward a sensor localization algorithm based on the integration of DV-Hop algorithm and Steffensen iteration. The paper first uses DV-Hop algorithm for rough localization of wireless sensor nodes; corrects the localization results of DV-Hop algorithm by using Steffensen iteration method, and achieves precise localization. Simulation results show that DVHop-Steff algorithm has improved the localization accuracy and has better practical values. 


\section{References}

[1] W. S. Jun, Z. Peng and Y. H. Bing, "Node Localization Problem in Wireless Sensor Networks", Computer Science, vol. 31, no. 10, (2004), pp. 35-38.

[2] Y. Xiao,W. Z. You and X. X. Liang, "A Centroid Localisation Algorithm Based on Secondary Anchor for Wireless Sensor Network", Computer Applications and Software, vol. 30, no. 6, (2013), pp.116-120.

[3] W. Wang, V. Srinivasan, B. Wang and K Chua, "Coverage for target localization in wireless sensor networks", IEEE Transactions on Wireless Communications, vol. 7, no. 2, (2008), pp. 667-676.

[4] J. WeiWei and L. Zhong, "Study on the Application of DV-Hop Localization Algorithms to Random Sensor Networks", Journal of Electronics \& Information Technology, vol. 30, no. 4, (2008) pp. 970-974.

[5] B. L. Zhang, F. Q. Yu and D. Lui, "Localization scheme for wireless sensor networks using directional antenna", 1EEE Transactions on Consumer Electronics, vol. 56, no. 4, (2010), pp. 2208-2216.

[6] S. Lee, E. Kim, C. Kim, et a1., "Localization with a mobile beacon based on geometric constraints in wireless sensor networks", IEEE Transactions on Wireless Communications, vol. 8, no. 12, (2009), pp. 5801-5805.

[7] Q. Fang, J. Gao and L.Guibas, "Locating and bypassing routing holes in sensor networks", Journal of Mobile Networks and Applications, vol.11, no. 2, (2006), pp. 187-200.

[8] N. Zhen, L. Y. Jie, S. Y. Fei and W. S. Cha, "An improved and modified localization algorithm based on DV-Hop", Transducer and Microsystem Technologies, vol. 29, no. 8, (2010), pp. 31-33.

[9] M. R. Ze, Y. Z. Jun and L. H. Tao, "A range-free localization algorithm for wireless sensor networks", Transducer and Microsystem Technologies, vol. 30, no. 30, (2011),pp. 131-134.

[10] C. Dun and C. Z. Qi, "New localization algorithm for wireless sensor networks", Computer Engineering and Applications, vol. 48, no. 8, (2012), pp.116-118.

[11] C. X. Zhou, L. M. Hua and L. J. Hua, "Improvement of node localization in wireless sensor network based on particle swam optimization", Journal of Computer Applications, vol. 30, no. 7, (2010), pp. 1736-1738.

[12] L. Mudong, X. Wei and L. Qing, “An Improved ABC-Based Node localization algorithm for wireless sensor networks", Proceedings of International Conference on Wireless Communications, Networking and Mobile Computing (WICOM), ShangHai, vol. 12, no. 12, (2012), pp. 1-4.

\section{Authors}

Wang ZhiPing, (1978), male, master, associate professor, research direction: wireless sensor.

Chen Xuan, (1979), male, master, lecturer, research direction: information security, wireless sensor and cloud computing. 